\title{
Avaliação das condições de saúde bucal dos idosos em um município brasileiro
}

\author{
Silvio Rocha Corrêa da Silva e Aylton Valsecki Júnior
}

RESUMO O objetivo deste estudo foi avaliar as condições de saúde bucal em pessoas com 60 anos ou mais no Município de Araraquara, Estado de São Paulo, Brasil, no ano de 1998. Participaram do estudo 194 pessoas (91 institucionalizadas, com idade média de 73,6 anos, e 103 não-institucionalizadas, com idade média de 69,3 anos), que foram examinadas por um cirurgião-dentista, previamente treinado, para determinar a prevalência das principais doenças bucais. Os resultados mostraram um grande número de pessoas edentadas (72\% dos institucionalizados e 60\% dos não-institucionalizados) e de dentes extraídos (93 e 90\%, respectivamente), grande freqüência de bolsas periodontais (57 e 75\%) e de próteses inadequadas (80\% das próteses em pessoas institucionalizadas e $61 \%$ em pessoas não-institucionalizadas). Os resultados mostram o comprometimento da qualidade de vida de grande parte dessas pessoas e indicam a necessidade de maior atenção dos serviços públicos a esse grupo populacional.

A população brasileira vem envelhecendo de forma rápida desde o início da década de 60, quando a queda das taxas de fecundidade começou a alterar a estrutura etária da população. Em 2020, cerca de 9\% da população terá 60 anos ou mais, contra $5 \%$ em 1996 (1).

A velocidade com a qual esse processo vem ocorrendo deverá determinar grandes dificuldades. Numa situação na qual os idosos não encontram amparo adequado no sistema público de saúde e previdência, tem-se como conseqüência o acúmulo de seqüelas de doenças, o desenvolvimento de in-

\footnotetext{
1 Universidade Estadual Paulista (UNESP), Faculdade de Odontologia de Araraquara, Departamento de Odontologia Social. Correspondência e pedidos de separatas devem ser enviados a Silvio Rocha Corrêa da Silva no seguinte endereço: UNESP, Faculdade de Odontologia, Departamento de Odontologia Social, Rua Humaitá 1680, CEP 14801-903, Araraquara, SP, Brasil. E-mail: silvio@foar.unesp.br
}

capacidades e a perda de autonomia e qualidade de vida (2).

Nesse contexto, a odontologia geriátrica começa a ganhar espaço, mas seu desenvolvimento vai depender de sua habilidade em demonstrar que pode entender os problemas desse grupo populacional e ajudar a encontrar as soluções. O processo de envelhecimento da população também obriga os profissionais e serviços de saúde a estarem preparados para o trabalho com essas pessoas e a diferenciarem adequadamente os fatores próprios do processo de envelhecimento normal daqueles que provêm do meio ambiente (3).

Apesar de não existirem doenças bucais relacionadas diretamente à velhice, alguns problemas, como a diminuição da capacidade mastigatória, a dificuldade de deglutição, a secura na boca, as modificações no paladar e a perda de dimensão vertical (espaço medido da base do nariz à ponta do queixo quando os dentes estão cerrados, com a boca fechada) têm efeitos cumulativos negativos e prejudiciais para o indivíduo. De acordo com Rosa et al. (4), os idosos sadios que têm seus dentes naturais e mantém uma estética e uma eficiência mastigatória aceitáveis podem e devem representar uma situação prevalente em cada comunidade.

Como no Brasil os programas dirigidos a esse grupo populacional ainda são raros, as poucas pesquisas epidemiológicas realizadas (4-9) mostram uma situação preocupante, pois, sem renda para a utilização de serviços privados e sem prioridade nos serviços públicos, os idosos apresentam grande quantidade de problemas bucais, como dentes extraídos, sextantes com doença periodontal, lesões da mucosa bucal e necessidade de próteses.

Este estudo foi realizado com o objetivo de descrever as condições de saúde bucal em uma amostra de pessoas com 60 anos ou mais, institucionalizadas e não-institucionalizadas, no 
Município de Araraquara, Estado de São Paulo, Brasil.

\section{MATERIAIS E MÉTODOS}

O estudo foi realizado no Município de Araraquara, durante o ano de 1998. Os dados foram coletados através de exame clínico em uma amostra de 194 pessoas com 60 anos ou mais.

Estabeleceu-se uma amostra de conveniência pela facilidade de acesso a essas pessoas. Assim, compuseram a população de estudo os indivíduos institucionalizados nos dois asilos do município e as pessoas inscritas no programa do idoso desenvolvido em um centro de saúde municipal. Esse programa serve de referência para todo o município e presta assistência multidisciplinar curativa e de reabilitação às pessoas com 60 anos ou mais, que receberam, neste estudo, a denominação de não-institucionalizadas.

O critério utilizado para a seleção da amostra, tanto em institucionalizados como em não-institucionalizados, foi o estado funcional do indivíduo, ou seja, o grau de independência com o qual os indivíduos desempenhavam as funções do dia-a-dia dentro de seu contexto socioeconômico e cultural. Foram incluídas no estudo as pessoas classificadas como funcionalmente independentes e semidependentes, de acordo com a classificação de Rosa et al. (4).

Os exames foram realizados por um cirurgião-dentista com o uso de luz artificial, sonda exploradora, espelho bucal plano, sonda periodontal da Organização Mundial da Saúde (OMS) para medição do índice de necessidade de tratamento (community periodontal index of treatment needs, CPITN) e espátulas de madeira. A avaliação do CPITN baseou-se nos critérios e registros da OMS $(10,11)$, utilizando seis dentes índices para representar os seis sextantes da boca. As fichas clínicas, baseadas na ficha padronizada de levantamentos epidemiológicos preconizada pela OMS, foram preenchidas por uma auxiliar.

Para medir a prevalência da cárie dentária foi empregado o índice CPO$\mathrm{D}$ (12), que indica o número médio de dentes permanentes cariados, perdidos e restaurados por idade ou grupo etário. Os critérios de diagnóstico foram os preconizados pela OMS (10) quando da realização de levantamentos epidemiológicos.

A avaliação do uso e da necessidade de prótese removível foi realizada a partir de adaptação dos critérios utilizados no levantamento epidemiológico em saúde bucal, realizado no Brasil, pelo Ministério da Saúde, em 1986 (5). Foram considerados:

a) uso de prótese removível: presença ou não, no momento do exame, de prótese total ou parcial removível.

b) necessidade de prótese removível: presença de prótese total ou parcial mas em condições clínicas inadequadas e, portanto, necessidade de nova prótese; ausência de prótese removível em pessoas com indicação clínica para tal, com, no mínimo, quatro ou mais dentes indicados para extração ou já extraídos no mesmo maxilar.

Foram consideradas inadequadas as próteses quebradas, com dentes gastos ou com falta de um ou mais dentes.

A apuração dos resultados e a análise estatística dos dados foram realizadas através do programa estatístico Epi Info, versão 6.04b. Numa primeira etapa, realizou-se a distribuição de freqüência das variáveis de estudo para a confecção de tabelas. Os testes estatísticos realizados tiveram como objetivo determinar a existência de diferenças significativas entre os dois grupos examinados e, para isso, foram utilizados os testes não-paramétricos de Mann-Whitney e o qui-quadrado $\left(\chi^{2}\right)$ de Pearson.

\section{RESULTADOS}

Os resultados referem-se às 194 pessoas com 60 anos ou mais examinadas. Destas, 91 viviam em asilos (institucionalizadas) e 103 não viviam em asilos (não-institucionalizadas).

A idade média encontrada foi de 71,4 anos, com os institucionalizados apresentando, em média, 73,6 anos, e os não-institucionalizados, 69,3 anos. Nos dois grupos examinados, $63 \%$ das pessoas eram do sexo feminino.

A falta de dentes foi observada em $72 \%$ das pessoas institucionalizadas e em $60 \%$ das não-institucionalizadas. Entre as pessoas dentadas institucionalizadas foram encontrados, em média, 3,15 dentes, enquanto os indivíduos dentados não-institucionalizados apresentavam, em média, 4,94 dentes.

$\mathrm{O}$ índice CPO-D (tabela 1) revelou que mais de $90 \%$ dos dentes já estavam perdidos. Os outros componentes do índice foram inexpressivos. Em média, cada pessoa possuía menos de dois dentes hígidos (1,09 entre os institucionalizados e 1,73 entre os não-institucionalizados). A análise do CPO-D mostrou diferença estatisticamente significativa entre os dois grupos apenas para número de dentes cariados $(P=0,006)$.

As próteses dentárias eram utilizadas por $63 \%$ das pessoas institucionalizadas e por $83 \%$ das não-institucionalizadas. A necessidade de prótese foi de $80 \%$ entre os não-institucionalizados e de $61 \%$ entre os institucionalizados (tabela 2), indicando que boa parte das próteses em uso não estava em condições clínicas satisfatórias e necessitava de substituição. Os dois grupos examinados apresentaram diferenças

TABELA 1. Distribuição do índice CPO-D e componentes segundo a condição funcional dos indivíduos examinados, Araraquara (SP), Brasil, 1998

\begin{tabular}{lccc}
\hline Componentes do índice & $\begin{array}{c}\text { Indivíduos } \\
\text { institucionalizados }\end{array}$ & $\begin{array}{c}\text { Indivíduos não- } \\
\text { institucionalizados }\end{array}$ & $P$ \\
\hline Dentes cariados & 1,31 & 1,01 & $0,006^{\mathrm{a}}$ \\
Dentes obturados & 0,75 & 1,98 & 0,450 \\
Dentes perdidos & 28,85 & 27,28 & 0,975 \\
CPO-D & 30,91 & 30,27 & 0,746 \\
\hline
\end{tabular}

a Estatisticamente significativo (teste de Mann-Whitney). 
TABELA 2. Distribuição de freqüência das condições protéticas segundo a condição funcional dos indivíduos examinados, Araraquara (SP), Brasil, 1998

\begin{tabular}{|c|c|c|c|c|}
\hline \multirow[b]{2}{*}{ Prótese } & \multicolumn{2}{|c|}{ Institucionalizados } & \multicolumn{2}{|c|}{ Não-institucionalizados } \\
\hline & $n$ & $\%$ & $n$ & $\%$ \\
\hline Não usa e não necessita & 1 & 1 & 5 & 5 \\
\hline Usa e não necessita de substituição & 17 & 19 & 35 & 34 \\
\hline Usa e necessita de substituição & 40 & 44 & 51 & 49 \\
\hline Não usa e necessita & 33 & 36 & 12 & 12 \\
\hline Total & 91 & 100 & 103 & 100 \\
\hline
\end{tabular}

estatisticamente significativas em relação ao uso $(P=0,001)$ e à necessidade $(P=0,004)$ de prótese, ou seja, constituíam, em termos estatísticos, amostras diferentes entre si.

Entre as pessoas institucionalizadas, a prótese estava em uso, em média, há 21 anos. Entre os não-institucionalizados, a média foi de 13,5 anos.

Dentre os itens do índice CPITN que indicam necessidade de tratamento, as bolsas periodontais foram o item de maior prevalência em ambos os grupos (tabela 3). Como a quantidade de dentes presentes era pequena, a média de sextantes excluídos do cálculo do índice foi elevada (3,76 entre os institucionalizados e 3,08 entre os não-institucionalizados).

\section{DISCUSSÃO}

Os resultados do presente estudo são representativos dos dois grupos de pessoas examinadas e não podem ser generalizados para toda a população idosa do município, pois os locais pesquisados atendem basicamente pessoas com baixa renda ou escolaridade.

Observa-se, nos dois grupos, que a condição clínica, de maneira geral, é

precária, segundo os vários indicadores utilizados. Porém, a condição clínica não é muito diferente da situação encontrada em outros estudos realizados no Brasil $(4,5,8,9)$ e em outros países (13-15). Além disso, os idosos institucionalizados apresentaram pior condição bucal do que os idosos nãoinstitucionalizados, o que já havia sido observado por outros autores $(4,16)$. Estatisticamente, mostrou-se que os institucionalizados constituíam um grupo diferente dos não-institucionalizados em termos de uso e necessidade de prótese e de dentes cariados.

A cárie dentária foi medida pelo índice CPO-D, que mostrou grande prevalência dessa doença. $\mathrm{O}$ número de dentes hígidos $(1,09$ entre os institucionalizados e 1,73 entre os nãoinstitucionalizados) e o percentual de pessoas edentadas (72 e $60 \%$ para institucionalizados e não, respectivamente) revelam não só a condição de muitas perdas dentárias, como o tipo de serviço odontológico prestado a essas pessoas, deixando claro que o tratamento restaurador falhou ou não chegou a existir.

Nos dois grupos, a quantidade de pessoas que não necessitavam do uso de uma prótese ou que já usavam um

TABELA 3. Porcentagem de indivíduos de acordo com a necessidade de tratamento e a condição funcional, Araraquara (SP), Brasil, 1998

\begin{tabular}{llllll}
\hline & \multicolumn{5}{c}{ Necessidade de tratamento conforme o CPITNa $(\%)$} \\
\cline { 2 - 6 } Condição funcional & 0 & 1 & 2 & 3 & 4 \\
\hline Institucionalizados & 0 & 9 & 33 & 38 & 19 \\
Não-institucionalizados & 0 & 0 & 25 & 45 & 30
\end{tabular}

a Community periodontal index of treatment needs. 0 = Sadio; 1 = sangramento gengival; 2 = cálculo dental; $3=$ bolsa periodontal rasa; 4 = bolsa periodontal profunda. aparelho considerado como clinicamente adequado foi menor em relação às pessoas que precisavam substituir a prótese que estava em uso ou começar a usá-la.

A grande quantidade de próteses que necessitavam de substituição, nos dois grupos examinados, decorre do fato de que esses aparelhos são utilizados durante vários anos, como pode ser visto pelo tempo médio pelo qual a prótese estava em uso (21 e 13,5 anos entre institucionalizados e não, respectivamente), provavelmente sem acompanhamento profissional, prejudicando a mastigação dos alimentos, o consumo de uma dieta adequada e o aparecimento de lesões na mucosa bucal, como demonstrado por Rosa et al. (4) e Birman et al. (6).

Sobre a condição periodontal, observou-se que a aplicação do índice CPITN foi prejudicada pela grande quantidade de dentes perdidos e de pessoas edentadas. Nos exames realizados nas instituições, o índice não foi aplicado em $77 \%$ dos indivíduos. Entre os não-institucionalizados, o percentual foi de $63 \%$. O principal problema encontrado foi a bolsa periodontal, especialmente entre os institucionalizados, embora não tenha sido notada diferença estatisticamente significativa entre os dois grupos.

Os dados clínicos sugerem que a qualidade de vida de grande parte dos participantes do estudo estava comprometida, de alguma maneira, pelas condições bucais e suas seqüelas, mesmo considerando que a qualidade de vida, segundo a OMS (17), deve ser avaliada a partir de vários critérios e levando em consideração não só a saúde física, mas também o estado psicológico, o nível de independência, os relacionamentos sociais, os fatores do meio ambiente e as crenças pessoais.

Os resultados deste estudo indicam a necessidade de maior atenção aos idosos por parte dos serviços públicos de saúde. Além da implantação de atendimento curativo e reabilitador, que tem alto custo, seria também necessário o desenvolvimento de ações preventivas e educativas, pois, como afirmam Vasconcellos e Amaral (18), as principais doenças da boca devem 
ser consideradas como doenças do comportamento e, conseqüentemente, do estilo de vida.

A promoção da saúde e a conseqüente mudança de comportamento são facilitadas pela educação. Segundo Mariño (19), todos os profissionais de saúde devem ser motivados pelo ci- rurgião-dentista a educar e assessorar seus pacientes no sentido de mostrarlhes os cuidados que devem ter com a boca e a necessidade de realizar, periodicamente, um auto-exame de tecidos moles.

A situação epidemiológica encontrada neste estudo indica que algo pre- cisa ser feito. Acreditamos que os recursos disponíveis possam ser distribuídos de maneira mais equilibrada, sem que se deixe de dar atenção a outros grupos prioritários, levando em conta a atual situação demográfica e epidemiológica do país.

\section{REFERÊNCIAS}

1. Fundação Instituto Brasileiro de Geografia e Estatística. Características demográficas e socioeconômicas da população. IBGE: Anuário Estatístico do Brasil; 1994. pp. 1-32.

2. Chaimowicz F. A saúde dos idosos brasileiros às vésperas do século XXI: problemas, projeções e alternativas. Rev Saude Publica 1997;31:184-200.

3. Pérez EA, Mariño R, Gillespie G, González R. Estado de la educación en gero-odontología en la América Latina: hallazgos de una encuesta. Educ Med Salud 1992;26:426-429.

4. Rosa AGF, Castellanos RA, Pinto VG. Saúde bucal na terceira idade. Rev Gaucha Odontol 1993;41:97-102.

5. Brasil, Ministério da Saúde. Levantamento epidemiológico em saúde bucal: Brasil, zona urbana, 1986. Brasília: Centro de Documentação do Ministério da Saúde; 1988.

6. Birman EG, Silveira FRX, Sampaio MCC. Prevalência de lesões da mucosa bucal em pacientes geriátricos. Rev Fac Odontol Zona Leste 1991;31:17-25.

7. Neves LHM. Câncer de boca: mortalidade entre os residentes no Estado de São Paulo no período de março de 1979 a fevereiro de 1982 [tese de doutorado]. São
Paulo: Faculdade de Saúde Pública, Universidade de São Paulo; 1994

8. Watanabe MGC. Experiência de cárie de superfície radicular em adultos atendidos na Faculdade de Odontologia de Ribeirão Preto — USP, 1993-1994 [dissertação de mestrado]. São Paulo: Faculdade de Saúde Pública, Universidade de São Paulo; 1994.

9. Pereira AC, Castellanos RA, Silva SRC, Watanabe MGC, Queluz DP, Meneghim MC. Oral health and periodontal status in Brazilian elderly. Braz Dent J 1996;7: 97-102.

10. Organização Mundial da Saúde. Levantamento epidemiológico básico de saúde bucal: manual de instruções. 3a ed. São Paulo: Editora Santos; 1991.

11. Cutress TW, Ainamo J, Sardo-Infirri J. The community periodontal index of treatment needs (CPITN) procedure for population groups and individuals. Int Dent J 1987;37:222-233.

12. Chaves MM. Odontologia social. 3a ed. São Paulo: Editora Artes Médicas; 1986.

13. Bergman JD, Wright FAC, Hammond RH. The oral health of the elderly in Melbourne. Aust Dent J 1991;36:280-285.

14. Steele JG, Walls AWG, Ayatollhi SMT, Murray JJ. Major clinical findings from a dental survey of elderly people in three different English communities. Br Dent J 1996; 180:17-23.

15. Fukuda H, Shinsho F, Nakajima K, Tatara $K$. Oral health habits and the number of teeth present in Japanese aged 50-80 years. Community Dent Health 1997;14: 248-252.

16. Kalk W, Baat C, Meeuwissen JH. Is there need for a gerodontology? Int Dent J 1992; 42:209-116.

17. World Health Organization. The World Health Organization Quality of Life Assessment (WHOQOL): What is quality of life? World Health Forum 1996;17: 354-356.

18. Vasconcellos MCC, Amaral JS. A sociedade brasileira e a prevenção em saúde bucal. Rev Odontol Univ São Paulo 1992; 6:133-139.

19. Mariño R. La salud bucodental de los ancianos, realidad, mitos y posibilidades. Bol Oficina Sanit Panam 1994;116: 419-426.

Manuscrito recebido em 13 de outubro de 1999. Aceito em versão revisada em 16 de maio de 2000 .

ABSTRACT This study assessed the oral health of individuals 60 years or older in the city of Araraquara, São Paulo, Brazil, in 1998. Of the 194 people who participated in the study, 91 of them were institutionalized and had an average age of 73.6 years, and 103 were not institutionalized and had an average age of 69.3 years. The study participants were examined by a previously trained oral surgeon who determined the prevalence of the most common oral health problems. The results revealed a large number of edentulous individuals ( $72 \%$ of those institutionalized and $60 \%$ of the noninstitutionalized participants) and many persons with extracted teeth $(93 \%$ and $90 \%$, respectively), as well as a high frequency of periodontal pockets ( $57 \%$ and $75 \%$, respectively) and of inadequate dentures ( $80 \%$ and $61 \%$, respectively). Our results show reduced quality of life for a large proportion of these older individuals, and also indicate that public health services should pay greater attention to this population group. 